\title{
Three-dimensional perspective drawing of two-dimensional functions with microcomputers
}

\author{
MIGUEL A. GARCÍA-PÉREZ \\ Universidad Complutense, Madrid, Spain
}

\begin{abstract}
In this paper, we present a BASIC program for Apple II series computers that allows the user to obtain perspective drawings of two-dimensional functions of known analytical expression. Also, we outline a procedure for obtaining perspective representations of two-dimensional arrays of data using the same program. The program should run on any computer with graphics mode with only slight modifications in the graphics commands.
\end{abstract}

Representing three-dimensional (3-D) data in graphical form is becoming a more useful and necessary tool for visual scientists. 3-D plots are very useful aids in showing, for example, receptive fields and modulation transfer functions (as in Daugman, 1980), and in representing certain classes of experimental results (as in Daugman, 1984). These types of 3-D perspective drawings are usually produced by means of plotters controlled through special software. As an alternative, these graphics can also be represented in a photograph-like fashion using the graphics capability of most microcomputers and printers, the result being a gray-scale graphic in which the $z$-axis (height) is represented by luminance. Two similar approaches for accomplishing this task have been published recently in this journal (Harris, 1984; White, Brussell, Williams, \& Ròg, 1984). However, gray-scale graphics can only be taken as coarse representations, because most of the peculiarities in the data will not be noticeable due to our low ability to discriminate small luminance differences in high-contrast images.

This paper presents a BASIC program that creates a perspective drawing similar to those produced by plotters. The program works by drawing the cross-sectional profile of the two-dimensional (2-D) function along the $x$ direction at several equally spaced planes at the $y$ direction, and leaving hidden segments undrawn. Thus, the analytic expression of the surface must be known. An extension is outlined below that allows the user to plot the surface described by a 2-D array of data with unknown analytic form. The program is written in AppleSoft BASIC for an Apple II+ computer, but it will work with only slight modifications on any microcomputer having

This work was supported by the Universidad Complutense and by a grant from the Fundación Ramón Areces for a research project entitled "Visión Artificial y Visión Humana: Aplicaciones a la Percepción Visual en Robótica." I thank José san Antonio for giving me access to the laser writer used to produce Figures 2-5.

Address correspondence to Miguel A. Garcia-Pérez, Laboratorio de Visión, Facultad de Psicologia, Universidad Complutense, Campus de Somosaguas, 28023 Madrid, Spain. a graphics mode. The 3-D plot is produced on the monitor screen, but it can easily be printed and/or stored on disk with the help of suitable commands that differ from one microcomputer to another. ${ }^{1}$

\section{DESCRIPTION OF THE PROGRAM}

The program, shown in Figure 1, has two parts: (1) a number of parameters related to screen resolution and graphic format (lines 10-100), and (2) the implementation of the drawing algorithm (lines 110-300), which requires the $z$-coordinate to be expressed in line 200 as a function of the $x$ - and $y$-coordinates. The printed output of this program is shown in Figure 2. Some of the parameters in Part 1 of the program are of fundamental importance either to modify the relative scale of the plot or to adapt the program so as to match the resolution of the graphic screen. Thus, the number representing the largest addressable coordinate in the $x^{\prime}$ - and $y^{\prime}$-directions ${ }^{2}$ must be assigned, respectively, to variables XR and YR in line 10. Line 20 defines a square window over which the function is to be drawn. Variable X1 defines the lower $x$-coordinate in this window; variable $\mathrm{X} 2$ defines the upper $x$-coordinate; similarly, variables $\mathrm{Y} 1$ and $\mathrm{Y} 2$ define the lower and upper $y$-coordinates. Thus, line 20 in Figure 1 defines a square window with four units per side and whose center is at the origin of the $x y$ plane. Variables OX and OY in line 30 define the $x^{\prime}$ - and $y^{\prime}-$ coordinates, respectively, in the screen plane corresponding to the point at coordinates $(\mathrm{X} 1, \mathrm{Y} 1)$ in the real plane, where $\mathrm{X} 1$ and $\mathrm{Y} 1$ are the above-mentioned values. Variable LL, also in line 30, defines the length, in $x^{\prime}$-axis units (dots), of the segment that represents a distance of X2-X1 units in the real plane. The last variable in line 30, variable $\mathrm{PH}$, represents the number of dots per unit length in the screen representation of the vertical, $z$, axis. For the drawing to have the highest degree of precision, given the available graphic resolution, variable $O X$ should be assigned a value of zero, and variable $L L$ a value equal to the greater integer lower than $(X R+1) / 1.47$, although 


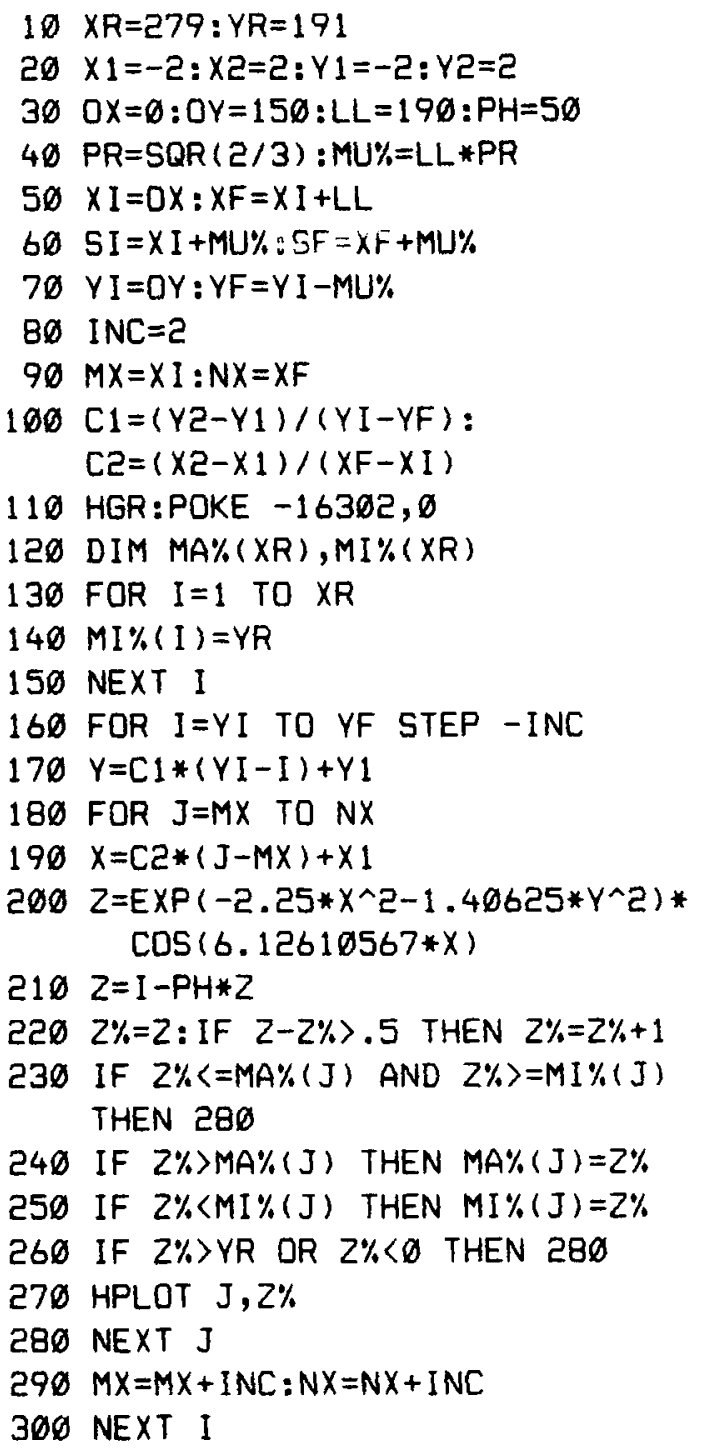

Figure 1. BASIC program to produce 3-D perspective drawings of 2-D functions. The code is explained in the text.

in some cases it might be increased by one or two units depending on the value assigned to variable INC in line 80. Variable $\mathrm{OY}$ allows the user to draw the picture at different vertical displacements on the screen plane so that surfaces of different heights (positive- as well as negative-valued) can fit completely on the screen. Rescaling the $z$-axis representation by changing the value of variable $\mathrm{PH}$ will also help to accomplish this task. Variables XI, XF, SI, SF, YI, and YF in lines 50-70 are the $x^{\prime}$ - and $y^{\prime}$-coordinates at the corners of the $x y$ window: upper left, (SI,YF); upper right, (SF,YF); lower left, (XI,YI); lower right, (XF,YI). The expressions that define each variable should not be modified for they depend on the value of the previous parameters. The user can interrupt program execution as drawing begins in order to check whether these variables are within limits and, if this is not the case, then the previous parameters (variables LL and OY in most cases) can be altered so that the whole $x y$ window fits onto the screen. Variable INC in line 80 defines the spacing (number of dot-lines plus one) between successive sections at corresponding $y$-values, and it also serves as the number of dots that each cross-sectional profile is shifted to the right with respect to the previously drawn profile. Finally, line 100 defines the two constants required to map the $x y$ window defined in line 20 onto the screen region defined in lines 50-70.

With regard to the second part of the program, commands in line 110 are AppleSoft-specific and they serve to set full-screen graphics mode. ${ }^{3}$ The arrays dimensioned in line 120 and both the explicit assignment of YR to all the elements of array MI\% (lines 130-150) and the implicit assignment of 0 to all the elements of array MA\% are the basis for the hidden points algorithm. Array MA\% stores, for every $x^{\prime}$-coordinate, the value of the highest $y^{\prime}$-coordinate at which a point has been plotted; similarly, array MI\% stores the value of the lowest $y^{\prime}$-coordinate filled with a point for every $x^{\prime}$-coordinate. The initial assignments to the elements of these arrays are consistent with the way in which the arrays signal the existence of a hidden or visible region. The drawing algorithm starts at line 160 and ends at the end of the program. Although the algorithm is quite simple, one can better understand it after seeing the program proceed in plotting dots on the screen. As the program runs, the picture is drawn from bottom to top and from left to right. The I-loop starting at line 160 controls the regular sampling of the $y^{\prime}$-axis, and line 170 calculates the particular $y$-value at that point, along which the one-dimensional profile of the function is to be plotted. The J-loop that starts at line 180 controls the position along the $x^{\prime}$-axis, and line 190 calculates the $x$-value at each point. Then line 200 obtains the value at the corresponding point of the function to be drawn, and lines 210-220 determine the $y^{\prime}$-coordinate at which the point should be plotted. Before plotting each dot, line 240 checks whether the point is hidden. As a matter of fact, all points whose $y^{\prime}$-coordinate is between the highest and the lowest coordinates already addressed at the particular $x^{\prime}$-coordinate (stored in arrays MA\% and MI\%, respectively) should be considered hidden. If this turns out to be true, the point is not plotted. Otherwise, the arrays are updated (lines 240-250), and if the $y^{\prime}$-coordinate is addressable (line 260), then the point is finally plotted (line 270). The HPLOT command in line 270 is also AppleSoft-specific and it is equivalent to the PSET command in IBM BASICA. The program iterates until all the one-dimensional (1-D) profiles have been plotted and the location of each of these profiles is shifted upward by means of the control variable of the J-loop and displaced laterally to the right (line 290) in order to produce the perceptual effect of perspective. Variables MX and NX define the range of $x^{\prime}$ values for each cross-sectional profile; in line 90 , they take initial values at the bottom of 
the screen representation of the $x y$ window and they both increase in line 290 by an amount of INC from one iteration to another.

The right-hand side of the assignment in line 200 corresponds to an even-symmetric 2-D Gabor function of the form

$f(x, y)=\exp \left\{-\pi\left[a^{2}\left(x-x_{0}\right)^{2}+b^{2}\left(y-y_{0}\right)^{2}\right]\right\} \cdot \cos \left(2 \pi u_{0} x+\phi\right)$,

with parameters $x_{0}=0, y_{0}=0, a=0.846, b=0.669$, $u_{0}=0.975$, and $\phi=0$. Figure 2 shows the corresponding perspective drawing. The corners of the rhomboid over which the surface ascends, labeled A, B, C, and D in Figure 2, correspond to the upper left, upper right, lower left, and lower right corners, respectively, of the square window in the $x y$ plane. Given the parameters in the program in Figure 1 , the $x^{\prime}$ - and $y^{\prime}$-coordinates of points $\mathrm{A}$, $B, C$, and D are $(89,61),(279,61),(0,150)$, and $(190,150)$, respectively. The number of dots separating points $C$ and $D$ is 190 (the value of variable $L L$ ).

\section{ADDITIONAL FEATURES OF THE PROGRAM}

Full representations over the $x y$ window have been discussed. One might also be interested in showing the crosssectional profile of a given 2-D function. This can be done easily with a slight modification of the program. Adding the line

\section{IF $\mathrm{Y}<0$ THEN $\mathrm{Z}=0$ : GOTO 210}

and deleting the cosine term in line 200 results in the design shown in Figure 3. It represents the $x$-axis profile of the gaussian envelope of the Gabor function in Figure 2 after a longitudinal section has been applied at the plane $y=0$. The added line sets the $z$-coordinate to zero, thus preventing it from being calculated by the assignment in line 200 , for all points satisfying the condition stated. Longitudinal sections at any plane can be drawn by replacing $\mathrm{Y}<0$ with $\mathrm{Y}<\mathrm{K}$ in line 195 above, where $K$ is the desired plane (Y1<K< 12 ). The 1-D profile in the $y$-axis direction can also be obtained using

195 IF $X>K$ THEN $Z=0$ : GOTO 210.

As in the previous case, $K$ must be replaced by the desired

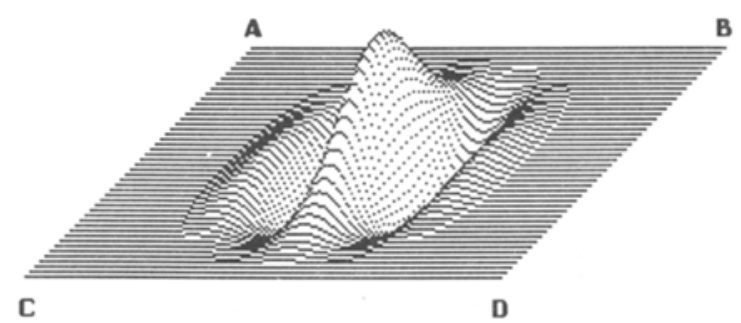

Figure 2. Even-symmetric 2-D Gabor function produced by the program in Figure 1. The $x$-axis is represented horizontally, the $y$-axis is tilted $45^{\circ}$, and the $z$-axis is represented vertically. Lower $x$-value is -2 ; upper $x$-value is 2 ; lower and upper $y$-values are the same as those of the $x$-axis. These characteristics are the same for the remaining figures in this paper.

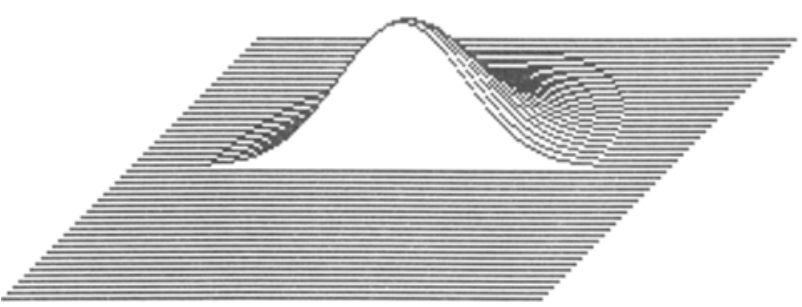

Figure 3. 1-D $x$-axis profile at the plane $y=0$ of a bivariate gaussian function. See text for parameters and program modifications needed to produce this plot.

value (with $\mathrm{X} 1<K<K 2$ ). Nonetheless, the $y$-axis profile will be distorted due to perspective, so it may be more useful to rotate the drawing by $90^{\circ}$ exchanging variables $X$ and $Y$ in line 200 and then drawing an $x$-axis profile, which will be the front view of the corresponding $y$-axis profile.

If the function has a range of negative values, as is the case for the Gabor function in Figure 2, it may be of interest to start drawing at the point where the profile is to be shown so that previous horizontal lines do not mask negative-valued parts. Adding the two lines

$$
\begin{aligned}
& 145 \text { MA } \%(\mathrm{I})=\text { YR } \\
& 195 \text { IF } \mathrm{Y}<0 \text { THEN } 280
\end{aligned}
$$

to the program in Figure 1 results in the design shown in Figure 4. The first of these lines acts as though a horizontal line had initially been drawn at the bottom of the screen, and it therefore prevents points from being plotted below the dotted line representing the crosssectional profile. The second added line prevents all points that satisfy the condition from being plotted. The previously made considerations regarding profiles at any given plane or profiles along the $y$-axis can be directly applied to this case too.

Up to now, we have been dealing with continuous functions. Discontinuous functions can also be represented with this program (in fact, Figure 3 was produced as a discontinuous function), but the result may be that some visible dots are interpreted as hidden and, therefore, are not plotted. This is shown in Figure 5a, which represents the Fourier magnitude spectrum of the cosine component of the Gabor function in Figure 2. To draw it, line 200 of the program is replaced by

$$
\begin{array}{rl}
200 & \mathrm{Z}=0: \mathrm{IF}(\mathrm{ABS}(\mathrm{X}-.975)<.05 \text { OR } \\
& \mathrm{ABS}(\mathrm{X}+.975)<.05) \text { AND } \\
& \mathrm{ABS}(\mathrm{Y})<.05 \text { THEN } \mathrm{Z}=0.5
\end{array}
$$

which is a close approximation to the function we want to draw, namely,

$$
f(x, y)=1 / 2\left[{ }^{2} \delta(x-0.975, y)+{ }^{2} \delta(x+0.975, y)\right],
$$

where ${ }^{2} \delta(\cdot, \cdot)$ represents the 2-D Dirac delta function (Bracewell, 1978). It can easily be seen in Figure 5a that the program fails in considering as hidden many dots that should be clearly visible. If the discontinuities of the function are known, this problem can be overcome. In fact, 
the program presented here draws the surface described by a function, and a point (or a line) of discontinuity means that the corresponding parts of the surface are not connected. Therefore, there are not vertical surfaces at the points of discontinuity, and the surface that lies behind the region of discontinuity in the same line of sight should be perfectly visible. If we know in advance that there should not be hidden dots in Figure $5 \mathrm{a}$, since the function is flat except for a vertical displacement of two regions, a program line (225 GOTO 260 ) can be used to skip the program segment that evaluates hidden dots; the result is shown in Figure 5b. This procedure does not have very wide applicability, however, and tailored methods would have to be designed for each particular case.

The program presented here covers the first of the two needs discussed at the beginning of the paper, that is, drawing 2-D functions in perspective view. With regard to plotting 2-D data arrays of arbitrary dimensions, the main problem arises because the data points do not usually exist at all of the coordinates in the $x y$ plane that are considered by the drawing algorithm. This problem, however, can be overcome using several procedures. One procedure consists of obtaining an interpolating polynomial (Atkinson, 1978) for each row in the array and representing it by means of this program. Repeating the procedure for every row achieves the desired perspective drawing. As an alternative, the surface to be plotted can be obtained by fitting a 2-D function to the data points (Cuyt \& Verdonk, 1985; Eden, Unser, \& Leonardi, 1986) and then drawing that function with the program presented here. A third possibility consists of arranging the data points, appropriately spaced, within a larger 2-D array representing the $x y$ window to be considered, and then lowpass filtering that array convolving it with the sinc function (Bracewell, 1978). The contents of the filtered array thus obtained will then represent the $z$-coordinate at each $(x, y)$ point, and that array can easily be plotted with this program if care has been taken to set the dimensions of the array exactly to the number of points that the program requires. Each method will yield different, but perhaps similar, results and the choice among them would probably depend both on their general characteristics as well as on the particular features in the original data. The comparative study of these methods has not been addressed

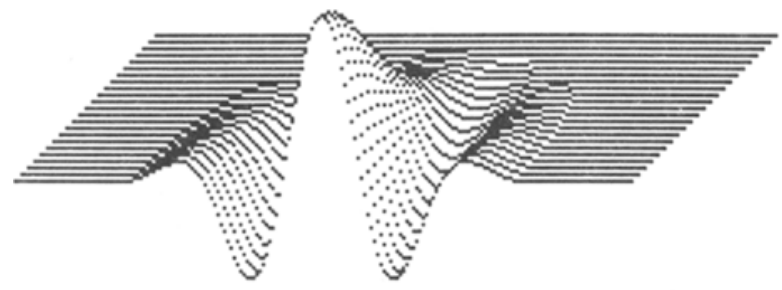

Figure 4. 1-D $x$-axis profile at the plane $y=0$ of the function in Figure 2. Changes in the program that lead to this plot are explained in the text.
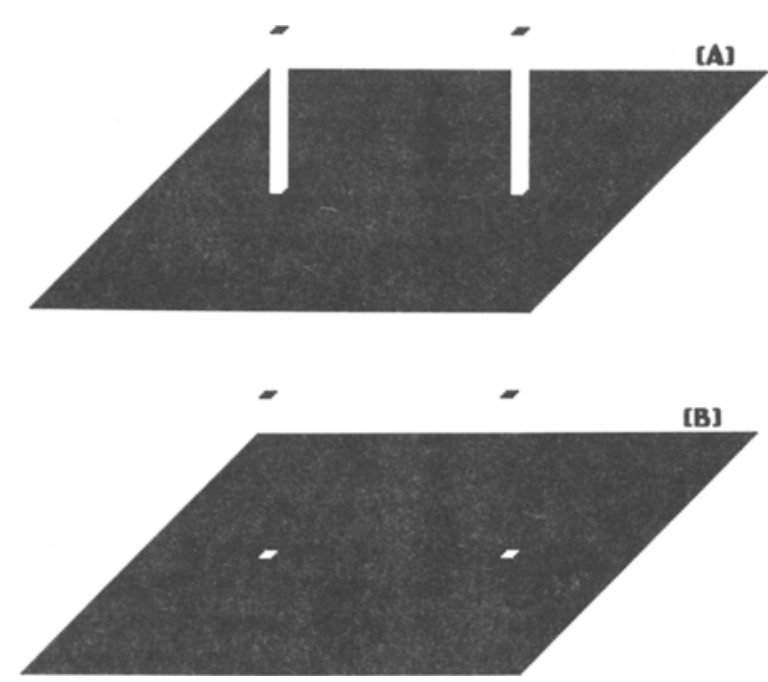

Figure 5. Fourier magnitude spectrum of a sine-wave grating with zero mean luminance, unit contrast, vertical orientation, and spatial frequency of 0.975 cycles per unit length. In this case, axes must be interpreted as the frequency dimensions $u$ and $v$, instead of the spatial dimensions $x$ and $y$. In practice, delta functions have been plotted as little squares with height equal to their area. To produce these plots, variable INC was set to 1 and variable $\mathbf{P H}$ was set to 120. (a) The failure of the program in considering all visible dots. (b) The performance of the program after skipping the lines that evaluate hidden dots.

to the extent required. Thus a final optimum solution cannot be presented as yet.

\section{REFERENCES}

AtKInson, K. E. (1978). An introduction to numerical analysis. New York: Wiley.

BRACEWELL, R. N. (1978). The Fourier transform and its applications. New York: McGraw-Hill.

CUYT, A. A. M., \& VERDONK, B. M. (1985). Multivariate rational interpolation. Computing, 34, 41-61.

Daugman, J. G. (1980). Two-dimensional spectral analysis of cortical receptive field profiles. Vision Research, 20, 847-856.

Daugman, J. G. (1984). Spatial visual channels in the Fourier plane. Vision Research, 24, 891-910.

Eden, M., UnSER, M. , \& LeONARDI, R. (1986). Polynomial representation of pictures. Signal Processing, 10, 385-393.

HARRIs, M. G. (1984). Printing gray-scale graphics using mean dot density. Behavior Research Methods, Instruments, \& Computers, 16, 570-571.

White, C. W., Brussell, E. M., Willuams, T. T., \& Ròg, S. J. (1984). Gray-scale graphics using dot matrix printers. Behavior Research Methods, Instruments, \& Computers, 16, 273-276.

\section{NOTES}

1. For the program in Figure 1, most users of Apple computers will know that the picture created can be saved to disk using the program line

$$
\text { PRINT CHR\$(4);"BSAVE ";F\$;“,A\$2000,L\$2000" }
$$

and any saved picture can be retrieved by the program line PRINT CHR\$(4);“BLOAD ";F\$;",A\$2000”, 
where $\mathrm{F} \$$ is a character variable containing the name of the file to be saved or retrieved.

2. We are dealing with two different 2-D planes: the screen plane where the dots are plotted invoking a pair of coordinates, and the real plane over which the function we want to draw is defined. To avoid confusion, $x^{\prime}$ and $y^{\prime}$ are used to refer to coordinates in the screen plane. In this plane, point $(0,0)$ is at the upper left corner. Coordinates in the real plane are referred to as $x$ and $y$, and $z$ is used for the third spatial coordinate (height).

3. For the drawing to have low distortion, the screen aspect ratio must be close to $1 / 1$. This means that if the program is to be run, for example, in BASICA on an IBM-PC (or compatible), line 110 should be replaced by

\section{CLS:KEY OFF:SCREEN 1}

rather than by

\section{CLS:KEY OFF:SCREEN 2}

since, in this microcomputer, low resolution yields a better aspect ratio.

(Manuscript received November 19, 1985; revision accepted for publication October 31,1986 .) 